\title{
Riemann Surface Structure for a Curved Surface with Punctured Features
}

\author{
Ramya Deepak Shetty ${ }^{1}$, Indira Narayana Swamy ${ }^{2}$ \& Govind R Kadambi ${ }^{3}$ \\ Ramaiah University of Applied Sciences, University House, Gnanagangothri Campus, New \\ BEL Road, M S R Nagar, Bangalore, Karnataka, INDIA - 560054.
}

Email: ${ }^{1}$ ramyahshetty@gmail.com, ${ }^{2}$ indira.sr.seg@msruas.ac.in, ${ }^{3}$ pvc.research@msruas.ac.in

\begin{abstract}
In this paper, a generic procedure for the development and subsequent validation of the Riemann surface structure (RSS) for a punctured curved surface lying on a Riemann surface is discussed. The proposed procedure differs from the existing methods involving triangular meshes and rectangular grids that rely on induced patches on surfaces. This procedure can be applied to non-punctured surfaces as well as to surfaces with irregularly located punctures. Further, by defining appropriate transition functions, the proposed procedure eliminates the requirement for smooth transitions across the boundaries of adjacent patches. The analytic formulations of the RSS for an ellipsoid and a sphere are elaborated using the proposed procedure. Moreover, the RSS of a sphere defined through a family of conformal unit discs is proven equivalent to that defined by an existing method based on stereographic projection. This study proves that a smooth projection between the surface and (a subset of) the complex plane $\mathbb{C}$, can be remapped to the original surface.
\end{abstract}

Keywords: Riemann surface structure, Canonical curved surface, Holomorphic function and Transition functions.

2020 Mathematics Subject Classification: 32H02, 32G99

\section{Introduction}

Surface modeling plays a substantial role in image analysis, surface projection, and object recognition. In the field of image processing [2, Figure 1, p.2], 3D surface models are used to visualize the geometry of a surface. For instance, in medical imaging use cases, it is vital to examine the anatomy of the brain surface [9, p.670] and subsequently map functional imaging parameters [10]. Generally, in surface modeling, any curved surface in $\mathbb{R}^{3}$ is considered a real manifold, i.e., as a rectangular grid $[1, \mathrm{p} .184]$ or a triangulated mesh [5], [4, p.8433]. Surface parameterization of these models using existing methods [8, p.704],[11, p.3] requires a differentiable one-to-one mapping of 3D surfaces onto 2D constraints (such as meshes and grids), such that the numerical computation can be easily formed by the resulting models.

Most existing methods for analyzing surfaces rely on induced grids or patches on the defined curved surface, which only have a one-to-one correspondence rather than a bijective (one-to-one and onto) correspondence between the original and the grid-induced surfaces. Further, both [4, p.8431] and [5, p.188] did not consider surfaces with punctured points or smooth transitions between the adjacent grids across grid boundaries. Moreover, pre-processing is performed to restrict the overlapping region between meshes or grids. However, due to the challenges involved in smoothly deforming a surface with genus to a 2D structure, 


\section{R. D. Shetty, I. N. Swamy \& G. R. Kadambi / Riemann Surface Structure for a Curved Surface...}

the generic approach proposed in this study uses RSS on a complex manifold for a curved surface with punctured points to overcome the above mentioned limitations by defining smooth transition functions.

\subsection{Riemann Surface Structure}

An RSS is a collection of compatible complex coordinate charts on a curved surface covering the entire surface. A Riemann surface $M$ is a surface with a class of charts $\left(U_{\alpha}, \varphi_{\alpha}\right), \alpha \in \mathbb{N}$, such that $\varphi_{\alpha} \cdot \varphi_{\beta}^{-1}$ is a holomorphic homeomorphism, where $\varphi_{\alpha}$ maps the open subset $U_{\alpha}$ of $M$ to an open subset of $\mathbb{C}$.

A coordinate chart is a homeomorphic map $\varphi$ from an open subset of a topological space $\mathrm{M}$ to an open subset of $\mathbb{R}^{2}(\mathbb{C})$, and is represented as the ordered pair $(U, \varphi)$. Assuming that $\left(U_{1}, \varphi_{1}\right)$ and $\left(U_{2}, \varphi_{2}\right)$ are two charts for an RSS with $U_{1} \cap \quad U_{2} \neq \phi$, then, the transition map $g_{12}$ is defined by $g_{12}=\varphi_{1} \cdot \varphi_{2}^{-1}$. As $\varphi_{1}$ and $\varphi_{2}$ are both homeomorphisms, the transition map is also homeomorphic. Two charts on a topological space are compatible if the transition map is smooth. An atlas for a topological space $\tau$ is the collection $\left\{\left(U_{\alpha}, \varphi_{\alpha}\right): \alpha \in \mathbb{N}\right\}$ of pairwise compatible charts whose domains cover $\tau$. In a complex manifold, the transition maps are holomorphic and are defined from $\mathbb{C}$ to $\mathbb{C}$. The pair $\left(U_{\alpha}, \varphi_{\alpha}\right)$ for each $\alpha \in \mathbb{N}$ is a complex co-ordinate chart [6, Definition 1.6, p.2].

Let $X$ be a curved surface with punctured points, lying on a Riemann surface $M$. Moreover, a curved surface can be represented as a family of open conformal unit discs, such that the union of these conformal unit discs covers the curved surface. Let $V_{k}$ be the $k$-th conformal unit disc such that $X=\mathrm{U}_{k} V_{k}$. If $f_{k}: V_{k} \rightarrow \mathbb{C}, k \in \mathbb{N}$, is a holomorphic function, then $\left\{\left(V_{k}, f_{k}\right), k \in \mathbb{N}\right\}$ defines the RSS on $X$.

This paper presents an analytical formulation for the development of the RSS for a collection of conformal open unit discs covering the curved surface on a Riemann surface. The RSS includes a holomorphic function and compatibility conditions between every pair of coordinate charts.

The remainder of this paper is structured into four sections and outlined as follows. In Section 2, the construction of the RSS of a curved surface $X$ on a Riemann surface using the defined complex coordinate charts, holomorphic atlas, and compatibility conditions is described. In Section 3, the generation process of the RSS of a typical canonical curved surface, namely an ellipsoid, is defined. In Section 4, the RSS of a sphere is validated based on the developed methodology by comparing it with that based on an existing procedure using stereographic projection. Finally, Section 5 presents the conclusion.

\section{Construction of an RSS on a Curved Surface}

A surface that is not flat and has smooth bending is called a curved surface. In topology, a surface is a topological space where every point has an open neighborhood, and it is homeomorphic to an open subset of the Euclidean plane [3, p.195]. This section deals with the analytical formulations involved in developing the RSS for a curved surface $X(x, y, z)$.

\subsection{Formulation of conformal unit disc}

Any curved surface can be defined as a family of conformal open unit discs such that the union of these conformal unit discs covers the entire curved surface. Let $X$ be a curved surface with a function $z=$ $F(x, y)$. If $\left\{V_{i}, i=1,2, \cdots, n\right\}$ are the open conformal unit discs covering $X$, then $X=\cup_{i} V_{i}$, i.e.,

$$
X=V_{1} \cup V_{2} \cup \cdots \cup V_{p} \cup \cdots \cup V_{n}
$$

Let $p$ be the number of punctured points on the curved surface, with $p<n$. Let the positional co-ordinates of the $k$-th puncture be denoted by $\left(x_{k}, y_{k}, z_{k}\right), k=1,2, \ldots, p$. For $k=1,2, \ldots, n, V_{k}$ are open conformal unit discs whose centers are the punctured points $\left(x_{k}, y_{k}, z_{k}\right)$ on the curved surface. By fixing $\left(x_{k}, y_{k}, z_{k}\right)$ on the curved surface, for all $(x, y, z) \in V_{k}$, the following equations hold. 


$$
\begin{aligned}
& \sqrt{\left(x-x_{k}\right)^{2}+\left(y-y_{k}\right)^{2}}<1 \\
& V_{k}=\left\{(x, y, z): d\left((x, y)-\left(x_{k}, y_{k}\right)\right)<1\right\}
\end{aligned}
$$

\subsection{Bi-holomorphic function}

Theorem 2.1 Let $f_{k}(x, y, z)=u+i v, \forall(x, y, z) \in V_{k}-\left\{\left(x_{k}, y_{k}, z_{k}\right)\right\}$

such that $(x, y, z),\left(x_{k}, y_{k}, z_{k}\right)$, and $(u, v, 0)$ are in the same line. Then $f_{k}, k=1,2, \ldots, n$, is a bijective bi-holomorphic function.

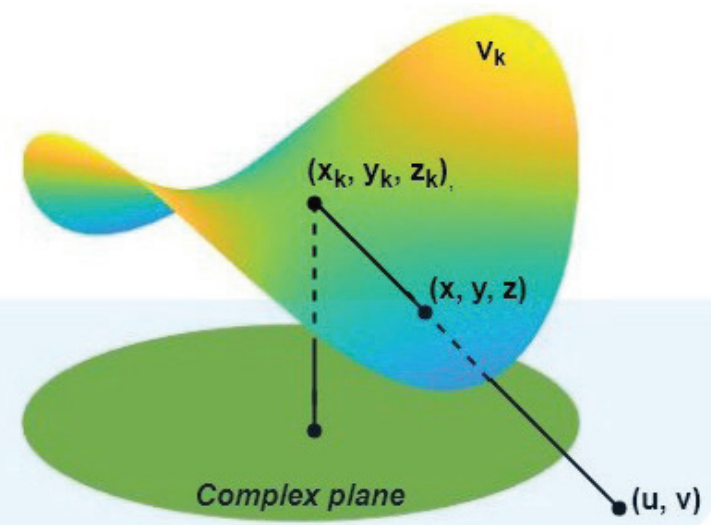

Proof.

Figure 1: Holomorphic function from $V_{k}$ to complex plane $(\mathbb{C})$

From Fig. 1 , it can be seen that $\left(x_{k}, y_{k}, z_{k}\right),(x, y, z)$, and $f_{k}(x, y, z)=(u, v)$ lie in the same straight line. Using the property of a straight line,

$$
\begin{gathered}
\frac{u-x}{x-x_{k}}=\frac{v-y}{y-y_{k}}=\frac{-z}{z-z_{k}}=r \\
u=x+\left(x-x_{k}\right) r, v=y+\left(y-y_{k}\right) r \text { and } r=\frac{-z}{z-z_{k}} \\
u=x-z \frac{x-x_{k}}{z-z_{k}} \text { and } v=y-z \frac{y-y_{k}}{z-z_{k}} \\
f_{k}(x, y, z)=x-z \frac{x-x_{k}}{z-z_{k}}+i\left(y-z \frac{y-y_{k}}{z-z_{k}}\right)
\end{gathered}
$$

where $u=x-z \frac{x-x_{k}}{z-z_{k}}$ and $v=y-z \frac{y-y_{k}}{z-z_{k}}$

Differentiating $u$ with respect to $x$ and $y$,

Differentiating $v$ with respect to $x$ and $y$,

$$
\begin{aligned}
& u_{x}=1-\frac{z}{z-z_{k}}=\frac{-z_{k}}{z-z_{k}} \\
& u_{y}=0 \\
& u_{z}=-\left(x-x_{k}\right)\left[\frac{z-z_{k}-z}{\left(z-z_{k}\right)^{2}}\right]=z_{k} \frac{x-x_{k}}{\left(z-z_{k}\right)^{2}}
\end{aligned}
$$

$$
\begin{aligned}
& v_{x}=0 \\
& v_{y}=1-\frac{z}{z-z_{k}}=\frac{-z_{k}}{z-z_{k}} \\
& v_{z}=-\left(y-y_{k}\right)\left[\frac{z-z_{k}-z}{\left(z-z_{k}\right)^{2}}\right]=z_{k} \frac{y-y_{k}}{\left(z-z_{k}\right)^{2}}
\end{aligned}
$$

The Jacobian matrix for Eq. (2.5) is given by,

$$
\Delta=\left(\begin{array}{lll}
u_{x} & u_{y} & u_{z} \\
v_{x} & v_{y} & v_{z}
\end{array}\right)=\left(\begin{array}{lll}
\frac{-z_{k}}{z-z_{k}} & 0 & z_{k} \frac{x-x_{k}}{\left(z-z_{k}\right)^{2}} \\
0 & \frac{-z_{k}}{z-z_{k}} & z_{k} \frac{y-y_{k}}{\left(z-z_{k}\right)^{2}}
\end{array}\right)
$$


R. D. Shetty, I. N. Swamy \& G. R. Kadambi / Riemann Surface Structure for a Curved Surface...

$$
\begin{aligned}
& \Delta_{1}=\left(\begin{array}{ll}
\frac{-z_{k}}{z-z_{k}} & 0 \\
0 & \frac{-z_{k}}{z-z_{k}}
\end{array}\right) \\
& \left\|\Delta_{1}\right\|=\left|\begin{array}{ll}
\frac{-z_{k}}{z-z_{k}} & 0 \\
0 & \frac{-z_{k}}{z-z_{k}}
\end{array}\right| \\
& \left\|\Delta_{1}\right\|=\frac{z_{k}^{2}}{\left(z-z_{k}\right)^{2}} \\
& \Delta_{2}=\left(\begin{array}{ll}
\frac{-z_{k}}{z-z_{k}} & z_{k} \frac{x-x_{k}}{\left(z-z_{k}\right)^{2}} \\
0 & z_{k} \frac{y-y_{k}}{\left(z-z_{k}\right)^{2}}
\end{array}\right) \\
& \left\|\Delta_{2}\right\|=\left|\begin{array}{ll}
\frac{-z_{k}}{z-z_{k}} & z_{k} \frac{x-x_{k}}{\left(z-z_{k}\right)^{2}} \\
0 & z_{k} \frac{y-y_{k}}{\left(z-z_{k}\right)^{2}}
\end{array}\right|=-\frac{z_{k}^{2}\left(y-y_{k}\right)}{\left(z-z_{k}\right)^{3}} \\
& \Delta_{3}=\left(\begin{array}{ll}
0 & z_{k} \frac{x-x_{k}}{\left(z-z_{k}\right)^{2}} \\
\frac{-z_{k}}{z-z_{k}} & z_{k} \frac{y-y_{k}}{\left(z-z_{k}\right)^{2}}
\end{array}\right) \\
& \left\|\Delta_{3}\right\|=\left|\begin{array}{ll}
0 & z_{k} \frac{x-x_{k}}{\left(z-z_{k}\right)^{2}} \\
\frac{-z_{k}}{z-z_{k}} & z_{k} \frac{y-y_{k}}{\left(z-z_{k}\right)^{2}}
\end{array}\right|=\frac{z_{k}^{2}\left(x-x_{k}\right)}{\left(z-z_{k}\right)^{3}}
\end{aligned}
$$

According to Royden's theorem [7, Proposition 1, p.306], "Suppose that $F: \mathbb{R}^{n} \rightarrow \mathbb{R}^{m}$. Write $F=$ $\left(f_{1}, \ldots, f_{m}\right)$, where $f_{i}: \mathbb{R}^{n} \rightarrow \mathbb{R}$. If for all $i$ and $j, \frac{D f_{i}}{D x_{j}}$ is (defined and) continuous near $t$, then $F$ is differentiable at $\mathrm{t}$, and the matrix for $D F(t)$ is given by the Jacobian matrix at the point $t$. If the determinant of the Jacobian matrix is non-zero for every point in topological space $S$ in $\mathbb{R}^{n}$, then the function $f$ is differential everywhere in $S$."

Clearly, $\left\|\Delta_{1}\right\|,\left\|\Delta_{2}\right\|$ and $\left\|\Delta_{3}\right\|$ are non-zero by the definition of $f_{k}$. Therefore, $f_{k}$ is complex differentiable at every point $(x, y, z)$ in an open conformal unit disc $V_{k}$ punctured at $\left(x_{k}, y_{k}, z_{k}\right)$. Hence, $f_{k}$ is a holomorphic function on an open set $V_{k}$ for all $k \in \mathbb{N}$. This, implies that the family of conformal unit discs entirely covering the curved surface satisfies the property of a holomorphic atlas.

The evaluation of the inverse function of $f_{k}$ is required for the compatibility condition, which must be satisfied by the given curved surface, such that it can be referred to as an RSS.

To generate the inverse of $f_{k}, k \in \mathbb{N}$, we have,

$$
\begin{aligned}
\frac{x-x_{k}}{x_{k}-u} & =\frac{y-y_{k}}{y_{k}-v}=\frac{z-z_{k}}{z_{k}}=t \\
x & =x_{k}+\left(x_{k}-u\right) t \\
y & =y_{k}+\left(y_{k}-v\right) t \\
z & =z_{k}+z_{k} t
\end{aligned}
$$

By Eq. (2.16), the curved surface $X$ is given by,

$$
X=X(x, y, z)=X\left(x_{k}+\left(x_{k}-u\right) t, y_{k}+\left(y_{k}-v\right) t, z_{k}+z_{k} t\right)
$$

By solving Eq. (2.17), $t$ can be parameterized as a function of $u$ and $v$, i.e., $\sigma(u, v)=t$ :

$$
f_{k}^{-1}(u, v)=\left(x_{k}+\left(x_{k}-u\right) \sigma(u, v), y_{k}+\left(y_{k}-v\right) \sigma(u, v), z_{k}+z_{k} \sigma(u, v)\right)
$$

The function $f_{k}^{-1}$ defined in Eq. (2.18) is holomorphic using the Jacobian matrix described in Royden's theorem. The associated holomorphic atlas can be represented as a set $A$ denoted by

$$
A=\left\{\left(V_{1}, f_{1}\right), \ldots,\left(V_{i}, f_{i}\right), \ldots,\left(V_{p}, f_{p}\right), \ldots,\left(V_{n}, f_{n}\right)\right\} .
$$




\subsection{Compatibility condition}

The compatibility condition must be satisfied by the given curved surface, such that it can be referred to as an RSS. In Section 2.2, Royden's theorem has been used to prove that the functions $f_{k}$ and $f_{k}^{-1}$ are holomorphic for all $k \in \mathbb{N}$. This section discusses the composition operation of two holomorphic functions, namely $f_{k}$ and $f_{j}^{-1}$, which leads to the definition of the transition function.

Corollary 2.1 If $V_{k} \cap V_{j} \neq \varnothing$ for some $k, j \in \mathbb{N}$ and $(u, v)$ in $V_{k} \cap V_{j}$, then the transition function

$$
\begin{aligned}
& g_{j k}: \mathbb{C} \rightarrow \mathbb{C} \text { as } \\
& g_{j k}(u, v)=f_{j} \cdot f_{k}^{-1}(u, v), \forall(u, v) \in V_{k} \cap V_{j}
\end{aligned}
$$

is a holomorphic function.

Because the holomorphic functions $f_{k}, k \in \mathbb{N}$ referred to in Eq. (2.5) that are defined on the family of conformal unit discs on $X$ and satisfy the compatibility condition, the curved surface has the RSS defined through the family of ordered pairs $\left(V_{k}, f_{k}\right), k=1,2, \ldots, n$, as defined in Eq. (2.19).

\section{Riemann Surface Structure of Canonical Curved Surface}

The most common canonical forms of curved surfaces are the ellipsoid, hyperboloid, and paraboloid. Section 3.1 presents a detailed procedure for defining the RSS for an ellipsoid.

\subsection{Ellipsoid}

This section considers the analytical formulations involved in the development of the RSS for an ellipsoid. Any arbitrary point $(x, y, z)$ on an ellipsoidal surface of revolution is governed by the equation,

$$
\frac{x^{2}}{a^{2}}+\frac{y^{2}}{b^{2}}+\frac{z^{2}}{c^{2}}=1
$$

where $a, b$, and $c$ are the semi-major, semi-minor, and semi-median axes, respectively. The geometrical profile of an ellipsoid in a $3 \mathrm{D}$ representation is shown in Fig. 2.

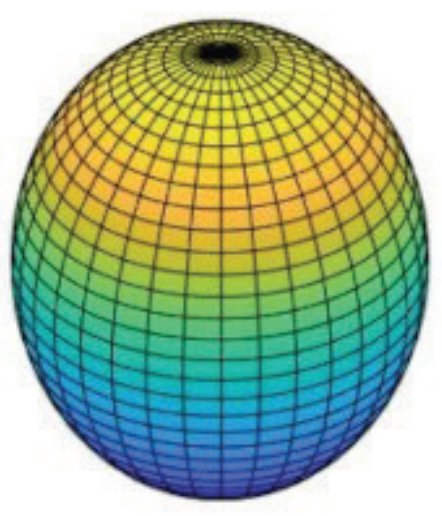

$\mathbf{a}<\mathbf{b}$

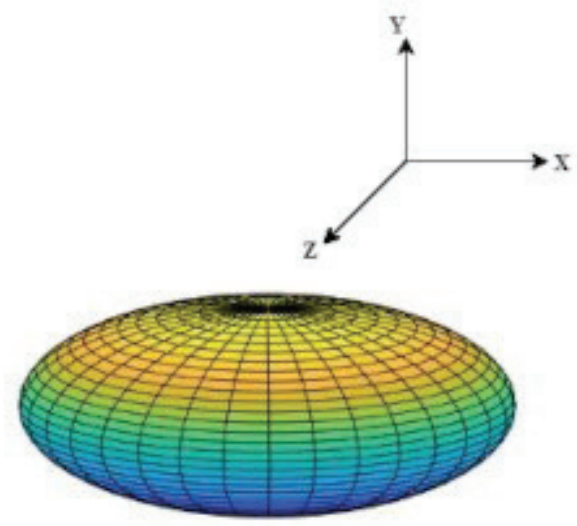

$\mathbf{a}>\mathbf{b}$

Figure 2: Ellipsoid with (i) $\mathrm{a}<\mathrm{b}$ and (ii) $\mathrm{a}>\mathrm{b}$

\subsubsection{Holomorphic function}

The functions $f_{k}, k=1,2, \ldots, n$, defined on the family of conformal unit discs on the ellipsoid are verified for their holomorphicity. Let $f_{k}: V_{k} \rightarrow \mathbb{C}$ such that

$$
f_{k}(x, y, z)=u+i v
$$

where $V_{k}$ is a conformal unit disc on the ellipsoid described in Section 2.1. From Fig. 1, $\left(x_{k}, y_{k}, z_{k}\right)$, $(x, y, z)$, and $f_{k}(x, y, z)=(u, v)$ lie in the same straight line. By the property of a straight line,

$$
\frac{u-x}{x-x_{k}}+\frac{v-y}{y-y_{k}}+\frac{-z}{z-z_{k}}=r
$$


R. D. Shetty, I. N. Swamy \& G. R. Kadambi / Riemann Surface Structure for a Curved Surface...

$$
f_{k}(x, y, z)=x-z \frac{x-x_{k}}{z-z_{k}}+i\left(y-z \frac{y-y_{k}}{z-z_{k}}\right)
$$

As stated in Section 2.2, Royden's theorem can be used to prove that $f_{k}$ defined in Eq. 3.4 is complex differential at every point $(x, y, z) \in V_{k}-\left\{\left(x_{k}, y_{k}, z_{k}\right)\right\}$ using the property of a Jacobian matrix. This, implies that the family of conformal unit discs that entirely cover the ellipsoid satisfies the property of a holomorphic atlas.

\subsubsection{Inverse of holomorphic function}

This section describes the evaluation of the inverse function of $f_{k}$ required for the compatibility condition. To generate the inverse of $f_{k}, k \in \mathbb{N}$, we have,

$$
\frac{x-x_{k}}{x_{k}-u}=\frac{y-y_{k}}{y_{k}-v}=\frac{z-z_{k}}{z_{k}}=t
$$

Which gives $x=x_{k}+\left(x_{k}-u\right) t, y=y_{k}+\left(y_{k}-v\right) t, z=z_{k}+z_{k} t$.

By Eq. (3.1), we have

$$
\begin{gathered}
\frac{\left(x_{k}+\left(x_{k}-u\right) t\right)^{2}}{a^{2}}+\frac{\left(y_{k}+\left(y_{k}-v\right) t\right)^{2}}{b^{2}}+\frac{\left(z_{k}+z_{k} t\right)^{2}}{c^{2}}=1 \\
\text { or, } \frac{x_{k}^{2}}{a^{2}}+\frac{\left(\left(x_{k}-u\right) t\right)^{2}}{a^{2}}+\frac{2 x_{k} t\left(x_{k}-u\right)}{a^{2}}+\frac{y_{k}^{2}}{b^{2}}+\frac{\left(\left(y_{k}-v\right) t\right)^{2}}{b^{2}}+\frac{2 y_{k} t\left(y_{k}-v\right)}{b^{2}}+\frac{z_{k}^{2}}{c^{2}}+\frac{\left(z_{k} t\right)^{2}}{c^{2}}+\frac{2 t z_{k}^{2}}{c^{2}}=1 \\
\text { or, } t^{2}\left[\frac{x_{k}^{2}}{a^{2}}+\frac{y_{k}^{2}}{b^{2}}+\frac{z_{k}^{2}}{c^{2}}-\frac{2 u x_{k}}{a^{2}}-\frac{2 v y_{k}}{b^{2}}+\frac{u^{2}}{a^{2}}+\frac{v^{2}}{b^{2}}\right]+2 t\left[\frac{x_{k}^{2}}{a^{2}}+\frac{y_{k}^{2}}{b^{2}}+\frac{z_{k}^{2}}{c^{2}}-\frac{u x_{k}}{a^{2}}-\frac{v y_{k}}{b^{2}}\right]=0 \\
\text { or, } t^{2}\left[1-\frac{2 u x_{k}}{a^{2}}-\frac{2 v y_{k}}{b^{2}}+\frac{u^{2}}{a^{2}}+\frac{v^{2}}{b^{2}}\right]+2 t\left[1-\frac{u x_{k}}{a^{2}}-\frac{v y_{k}}{b^{2}}\right]=0
\end{gathered}
$$

Which gives $t=\frac{2\left(\frac{u x_{k}}{a^{2}}+\frac{v y_{k}}{b^{2}}-1\right)}{\left(1-\frac{2 u x_{k}}{a^{2}}-\frac{2 v y_{k}}{b^{2}}+\frac{u^{2}}{a^{2}}+\frac{v^{2}}{b^{2}}\right)}$ as $t \neq 0$

$$
x=\frac{\left(2 u\left(1-\frac{y_{k}^{2}}{b^{2}}\right)-x_{k}\left(1+\frac{u^{2}}{a^{2}}-\frac{v^{2}}{b^{2}}\right)\right.}{\left(1-\frac{2 u x_{k}}{a^{2}}-\frac{2 v y_{k}}{b^{2}}+\frac{u^{2}}{a^{2}}+\frac{v^{2}}{b^{2}}\right)}, y=\frac{\left(2 v\left(1-\frac{x_{k}^{2}}{a^{2}}\right)-x_{k}\left(1+\frac{v^{2}}{b^{2}}-\frac{u^{2}}{a^{2}}\right)\right.}{\left(1-\frac{2 u x_{k}}{a^{2}}-\frac{2 v y_{k}}{b^{2}}+\frac{u^{2}}{a^{2}}+\frac{v^{2}}{b^{2}}\right)}, z=z_{k} \frac{\left(\frac{u x_{k}}{a^{2}}+\frac{v y_{k}}{b^{2}}-1\right)}{\left(1-\frac{2 u x_{k}}{a^{2}}-\frac{2 v y_{k}}{b^{2}}+\frac{u^{2}}{a^{2}}+\frac{v^{2}}{b^{2}}\right)}
$$

Therefore, $f_{k}^{-1}(u, v)=(x, y, z)$, where $x, y$, and $z$ are defined by Eq. (3.7), and it is holomorphic.

\subsubsection{Compatibility condition}

The compatibility condition must be satisfied by the given curved surface, such that it can be referred to as an RSS. In Sections 3.1.1 and 3.1.2, it was proved that the functions $f_{k}$ and $f_{k}^{-1}$ are holomorphic.

This section discusses the composition operation of two holomorphic functions, namely $f_{k}$ and $f_{k}^{-1}$, which leads to the definition of the transition function. If $(x, y, z) \in V_{i} \cap V_{j}$ for some $k, j \in \mathbb{N}$ with image $(u, v)$ in $\mathbb{C}$, then there exists a transition function defined by $g_{j k}: \mathbb{C} \rightarrow \mathbb{C}$ such that $g_{j k}(u, v)=$ $f_{j} \cdot f_{k}^{-1}(u, v), \forall(u, v) \in \mathbb{C}:$

$$
g_{j k}(u, v)=f_{j}\left(\frac{2 u\left[1-\frac{y_{k}^{2}}{b^{2}}\right]-x_{k}\left[1+\frac{u^{2}}{a^{2}}-\frac{v^{2}}{b^{2}}\right]}{\left[1-\frac{2 u x_{k}}{a^{2}}-\frac{2 v y_{k}}{b^{2}}+\frac{u^{2}}{a^{2}}+\frac{v^{2}}{b^{2}}\right]}, \frac{2 v\left[1-\frac{x_{k}^{2}}{a^{2}}\right]-x_{k}\left[1+\frac{v^{2}}{b^{2}}-\frac{u^{2}}{a^{2}}\right]}{\left[1-\frac{2 u x_{k}}{a^{2}}-\frac{2 v y_{k}}{b^{2}}+\frac{u^{2}}{a^{2}}+\frac{v^{2}}{b^{2}}\right]}, \quad z_{k} \frac{\left[\frac{u x_{k}}{a^{2}}+\frac{v y_{k}}{b^{2}}-1\right]}{\left[1-\frac{2 u x_{k}}{a^{2}}-\frac{2 v y_{k}}{b^{2}}+\frac{u^{2}}{a^{2}}+\frac{v^{2}}{b^{2}}\right]}\right.
$$

Where

and

$$
\begin{gathered}
g_{j k}(u, v)=P_{1}+i Q_{1} \\
P_{1}=\frac{\left[x_{j} z_{k}\left(\frac{u^{2}}{a^{2}}+\frac{v^{2}}{b^{2}}-1\right)-z_{j}\left(2 u\left(1-\frac{v y_{k}}{b^{2}}\right)-x_{k}\left(1+\frac{u^{2}}{a^{2}}-\frac{v^{2}}{b^{2}}\right)\right)\right]}{\left[z_{k}\left(\frac{u x_{k}}{a^{2}}+\frac{v y_{k}}{b^{2}}-1\right)-z_{j}\left(1-\frac{2 u x_{k}}{a^{2}}-\frac{2 v y_{k}}{b^{2}}+\frac{u^{2}}{a^{2}}+\frac{v^{2}}{b^{2}}\right)\right]} \\
Q_{1}=\frac{\left[y_{j} z_{k}\left(\frac{u^{2}}{a^{2}}+\frac{v^{2}}{b^{2}}-1\right)-z_{j}\left(2 v\left(1-\frac{u x_{k}}{a^{2}}\right)-y_{k}\left(1-\frac{u^{2}}{a^{2}}+\frac{v^{2}}{b^{2}}\right)\right)\right]}{\left[z_{k}\left(\frac{u x_{k}}{a^{2}}+\frac{v y_{k}}{b^{2}}-1\right)-z_{j}\left(1-\frac{2 u x_{k}}{a^{2}}-\frac{2 v y_{k}}{b^{2}}+\frac{u^{2}}{a^{2}}+\frac{v^{2}}{b^{2}}\right)\right]}
\end{gathered}
$$


Here, Eq. (3.8), which defines the transition function of an ellipsoid, is holomorphic. Therefore, the given holomorphic functions defined on the family of conformal unit discs on the ellipsoid satisfy the compatibility condition. The ellipsoid has the RSS defined through the family of ordered pairs $\left(V_{k}, f_{k}\right), k$ $=1,2, \ldots, n$, as defined in Eq. (2.19).

A similar procedure can be used to formulate the RSS for other canonical curved surfaces such as a hyperboloid of one sheet, a hyperboloid of two sheets, an elliptical paraboloid, and a hyperbolic paraboloid. The methodology as a pre-requisite requires curved surfaces to be defined as an algebraic expression, i.e., the surface must be expressed in the form of $z=F(x, y)$. Additionally, the methodology requires a smoothly overlapped region of conformal unit discs.

\section{Validation of an RSS with a Stereographic Projection}

In this section, we aim to validate the analytical expression for the RSS of a sphere derived through the family of conformal unit discs on the sphere by comparing it with the results obtained through a stereographic projection.

\subsection{RSS of an unit sphere}

Let $\mathbb{S}^{2}$ be a unit sphere in $\mathbb{R}^{3}$. Consider a set $A_{1}=\left\{\left(V_{1}, f_{1}\right),\left(V_{2}, f_{2}\right), \ldots,\left(V_{n}, f_{n}\right)\right\}$ for some natural number $n$, where $V_{k}, k \in \mathbb{N}$, are open discs with punctures at $\left(x_{k}, y_{k}, z_{k}\right)$ for $k \leq n$ on sphere $\mathbb{S}^{2}$, as shown in Fig. 3. Further, $f_{k}: V_{k} \rightarrow \mathbb{C}$ such that,

$$
\begin{aligned}
& f_{k}(x, y, z)=u+i v, \forall(x, y, z) \in V_{k}-\left\{\left(x_{k}, y_{k}, z_{k}\right)\right\} \\
& f_{k}(x, y, z)=x-z \frac{x-x_{k}}{z-z_{k}}+i\left(y-z \frac{y-y_{k}}{z-z_{k}}\right)
\end{aligned}
$$

As shown in Section 2.2, Royden's theorem can be used to prove that $f_{k}$ defined in Eq. (4.1) is complex differentiable at every point $(x, y, z) \in V_{k}-\left\{\left(x_{k}, y_{k}, z_{k}\right)\right\}$ using the property of a Jacobian matrix.

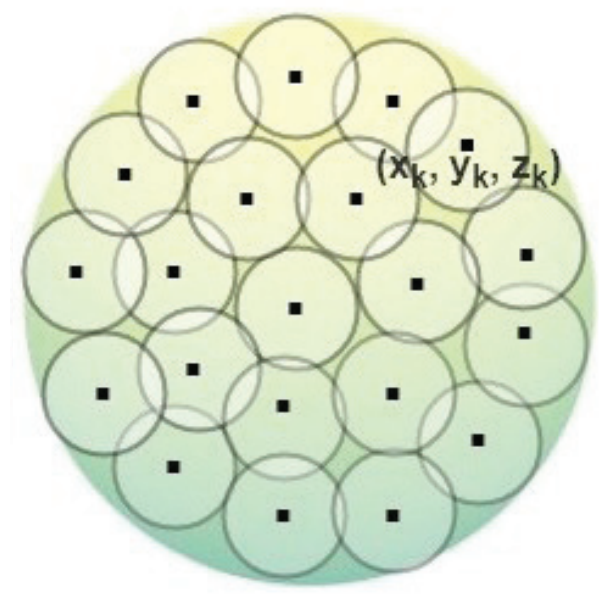

Figure 3: Family of conformal unit discs on a Riemann sphere

\subsubsection{Inverse of a function $f_{k}, k \in \mathbb{N}$}

$$
f_{k}^{-1}(u, v)=\left(\begin{array}{l}
\frac{x_{k}\left(v^{2}-u^{2}\right)-2 u\left(1+v y_{k}\right)}{1-2 u x_{k}-2 v y_{k}+u^{2}+v^{2}} \\
\frac{y_{k}\left(u^{2}-v^{2}\right)-2 v\left(1+u x_{k}\right)}{1-2 u x_{k}-2 v y_{k}+u^{2}+v^{2}} \\
z_{k} \frac{-1+u^{2}+v^{2}}{1-2 u x_{k}-2 v y_{k}+u^{2}+v^{2}}
\end{array}\right)^{T}
$$


R. D. Shetty, I. N. Swamy \& G. R. Kadambi / Riemann Surface Structure for a Curved Surface...

\subsubsection{Compatibility condition}

For each $(x, y, z) \in V_{k} \cap V_{j}, \forall k, j \in \mathbb{N}$, and $(u, v)$ in $\mathbb{C}$, the transition function $g_{j k}: \mathbb{C} \rightarrow \mathbb{C}$ is defined as $g_{j k}(u, v)=f_{j} \cdot f_{k}^{-1}(u, v)$

$$
g_{j k}(u, v)=P_{2}+i Q_{2}
$$

where

and

$$
\begin{aligned}
& P_{2}=\frac{\left[x_{j} z_{k}\left(u^{2}+v^{2}-1\right)-z_{j}\left(2 u\left(1-v y_{k}\right)-x_{k}\left(1+u^{2}-v^{2}\right)\right)\right]}{\left[z_{k}\left(u x_{k}+v y_{k}-1\right)-z_{j}\left(1-2 u x_{k}-2 v y_{k}+u^{2}+v^{2}\right)\right]} \\
& Q_{2}=\frac{\left[y_{j} z_{k}\left(u^{2}+v^{2}-1\right)-z_{j}\left(2 v\left(1-u x_{k}\right)-y_{k}\left(1-u^{2}+v^{2}\right)\right)\right]}{\left[z_{k}\left(u x_{k}+v y_{k}-1\right)-z_{j}\left(1-2 u x_{k}-2 v y_{k}+u^{2}+v^{2}\right)\right]}
\end{aligned}
$$

\subsection{Stereographic projection}

A unit sphere $\mathbb{S}^{2}$ in $\mathbb{R}^{3}$ with $N=(0,0,1)$ and $S=(0,0,-1)$ is shown in Fig. 4. Consider a set $A_{2}=$ $\left\{\left(U_{1}, \phi_{1}\right),\left(U_{2}, \phi_{2}\right)\right\}$, where $U_{1}=\mathbb{S}^{2}-\{N\}$ and $U_{2}=\mathbb{S}^{2}-\{S\}$.

$\phi_{1}: U_{1} \rightarrow \mathbb{C}$ (stereographic projection from $N$ ) such that,

$$
\phi_{1}(x, y, z)=\frac{x}{1-z}+i\left(\frac{y}{1-z}\right), \forall(x, y, z) \in \phi_{1}
$$

and $\phi_{2}: U_{2} \rightarrow \mathbb{C}$ (stereographic projection from $S$ ) such that

$$
\phi_{2}(x, y, z)=\frac{x}{1+z}+i\left(\frac{y}{1+z}\right), \forall(x, y, z) \in \phi_{2} .
$$

Therefore, for any points $P$ and $Q$ in $\mathbb{S}^{2}, \phi_{1}(P)$ is the intersection point of line $N P$ with the complex plane $\mathbb{C}$, and $\phi_{2}(Q)$ is an intersection point of line $S Q$ with the complex plane $\mathbb{C}$, as shown in Fig. 4

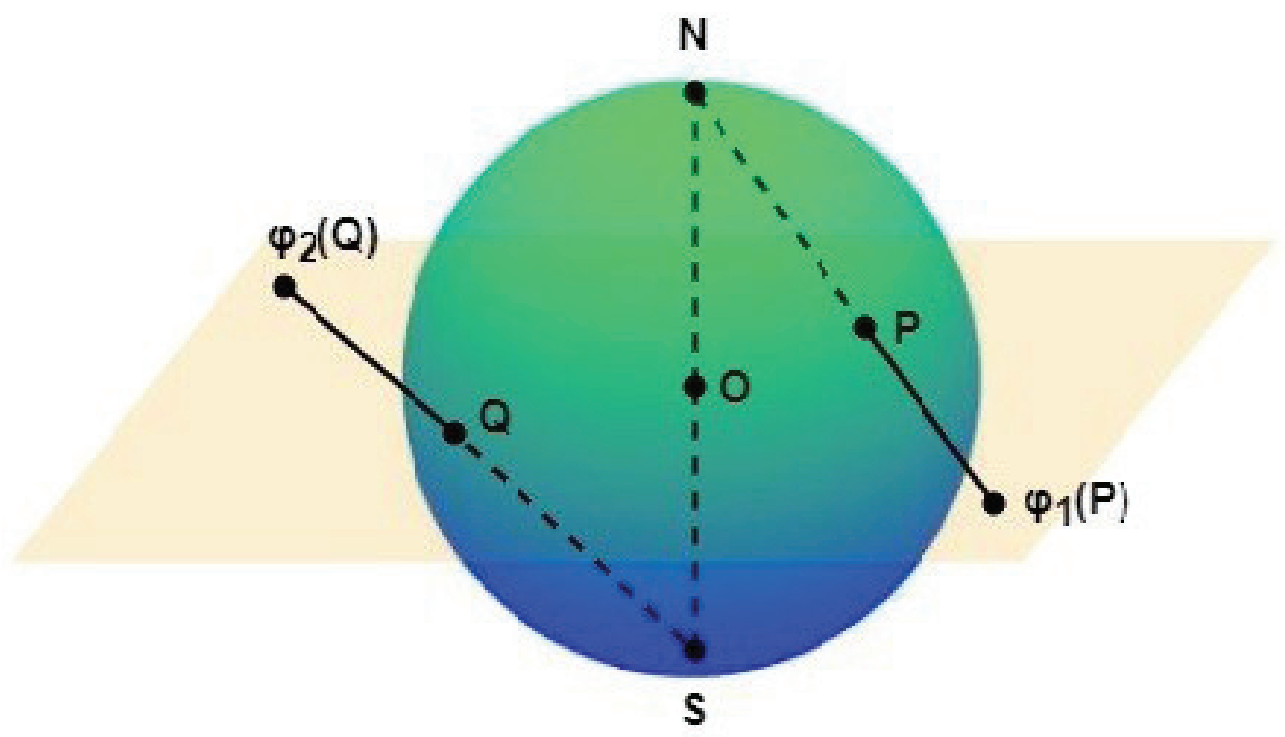

Figure 4: Stereographic projection of a Riemann sphere

\subsubsection{Compatibility condition}

Because $U_{1} \cap U_{2}=\mathbb{S}^{2}-\{N, S\}$ belongs to $\mathbb{S}^{2}, \phi_{1}\left(U_{1} \cap U_{2}\right)=\phi_{2}\left(U_{1} \cap U_{2}\right)=\mathbb{C}-\{(0,0)\}$, which shows that the domain has a puncture at $(\mathrm{u}, \mathrm{v})=(0,0)$ in $\mathbb{C}$. Therefore, the transition map $\sigma_{21}: \mathbb{C}-\{(0,0)\} \rightarrow \mathbb{C}-\{(0,0)\}$ is defined as $\sigma_{21}=\phi_{2} \cdot \phi_{1}^{-1}$. It can easily be verified that 


$$
\phi_{1}^{-1}(u, v)=\phi_{2}^{-1}(u, v)=\frac{1}{\left[1+u^{2}+v^{2}\right]}\left(2 u, 2 v,\left(-1+u^{2}+v^{2}\right)\right)
$$

where $\phi_{1}$ defined by Eq. (4.5) is holomorphic. Therefore,

$$
\sigma_{21}(u, v)=\frac{1}{u^{2}+v^{2}}(u+i v)
$$

is holomorphic. This $R S S$ defined through set $A_{2}$ on $\mathbb{S}^{2}$ is called the Riemann sphere.

Thus, $A_{1}$ is a holomorphic atlas defined by the family of conformal unit discs

$\left\{\left(V_{1}, f_{1}\right),\left(V_{2}, f_{2}\right), \ldots,\left(V_{n}, f_{n}\right)\right\}$, and $A_{2}$ is another atlas, defined by the stereographic projection $\left\{\left(U_{1}, \phi_{1}\right),\left(U_{2}, \phi_{2}\right)\right\}$. Two atlases of a topological space are said to be equivalent if every chart of one atlas is compatible with every chart of the other atlas. Subsequently, we discuss the equivalence of the two atlases $A_{1}$ and $A_{2}$ defined above.

For $z \neq 1$ (punctured at $N$ ), the transition function is,

$$
\begin{aligned}
\psi_{k 1}: \mathbb{C} \rightarrow \mathbb{C} \text { as } \psi_{k 1}(u, v) & =f_{k} \cdot \phi_{1}^{-1}(u, v) \\
\psi_{k 1}(u, v) & =P_{3}+i Q_{3}
\end{aligned}
$$

where

$$
P_{3}=\frac{\left[-2 u z_{k}+x_{k}\left(-1+u^{2}+v^{2}\right)\right]}{\left[\left(-1+u^{2}+v^{2}\right)-z_{k}\left(1+u^{2}+v^{2}\right)\right]} \quad \text { and } \quad Q_{3}=\frac{\left[-2 v z_{k}+y_{k}\left(-1+u^{2}+v^{2}\right)\right]}{\left[\left(-1+u^{2}+v^{2}\right)-z_{k}\left(1+u^{2}+v^{2}\right)\right]}
$$

Clearly, $\psi_{k 1}$ is holomorphic. For $z \neq-1$ (punctured at $S$ ), the transition function $\psi_{k 2}: \mathbb{C} \rightarrow \mathbb{C}$ is,

$$
\begin{aligned}
\psi_{k 2}(u, v)=f_{k} \cdot \phi_{2}^{-1}(u, v) & \\
\psi_{k 2}(u, v) & =P_{3}+i Q_{3}
\end{aligned}
$$

where $P_{3}=\frac{\left[-2 u z_{k}+x_{k}\left(-1+u^{2}+v^{2}\right)\right]}{\left[\left(-1+u^{2}+v^{2}\right)-z_{k}\left(1+u^{2}+v^{2}\right)\right]} \quad$ and $\quad Q_{3}=\frac{\left[-2 v z_{k}+y_{k}\left(-1+u^{2}+v^{2}\right)\right]}{\left[\left(-1+u^{2}+v^{2}\right)-z_{k}\left(1+u^{2}+v^{2}\right)\right]}$.

Thus, $\psi_{k 2}$ is also holomorphic. Therefore, the two atlases $A_{1}$ and $A_{2}$ are compatible and the RSS of a sphere defined through a family of conformal unit discs is equivalent to that defined through stereographic projection.

\section{Conclusion}

This paper introduced a generalized procedure for the development of the RSS for a curved surface by defining a family of conformal unit discs on the curved surface. The proposed method uses the holomorphic function defined for a family of conformal unit discs on a curved surface. Moreover, the proposed method can consider multiple unevenly distributed punctures on the curved surface. Further, the primary result was used to formulate a geodesic equation for a curved surface and establish the geodesic length function. In addition, the analytic formulations of the RSS for an ellipsoid and a sphere were elaborated using the developed procedure. Finally, the RSS of a sphere defined through a family of conformal unit discs was shown to be equivalent to that defined by an existing method using stereographic projection.

A generic procedure for the construction of a universal cover of an arbitrary, non-simply connected curved surface can overcome the need for the assumption that the entire surface must be simply connected. An elegant proof for the existence of a non-trivial equivalence between the universal cover and the fundamental group of the RSS of a curved surface was outlined, as well as validated using the uniformization theorem for simply connected Riemann surfaces. This would facilitate the determination of a geodesic 
R. D. Shetty, I. N. Swamy \& G. R. Kadambi /Riemann Surface Structure for a Curved Surface...

between any two points on the conformal unit disc. Moreover, a conformal mapping between the RSS and the curved surface will be defined to evaluate the geodesic between two points on the curved surface.

\section{Acknowledgment}

This manuscript was edited for English language by Elsevier Language Editing Services.

\section{References}

[1]. Carr N. A., Hoberock J., Crane K., and Hart J. C. (2006). Rectangular Multi-Chart Geometry Images. In Sheffer A. and Polthier K., editors, Symposium on Geometry Processing. The Eurographics Association.

[2]. Choi G. P., Leung-Liu Y., Gu X., and Lui L. M. (2020). Parallelizable global conformal parameterization of simply-connected surfaces via partial welding. SIAM Journal on Imaging Sciences. 13(3):1049-1083.

[3]. Jost J. (2006). Compact Riemann Surfaces: An Introduction to Contemporary Mathematics. Universitext. Springer Berlin Heidelberg.

[4]. Kimmel R. and Sethian J. A. (1998). Computing geodesic paths on manifolds. Proceedings of the National Academy of Sciences. 95(15):8431-8435.

[5]. Martínez D., Velho L., and Carvalho P. C. (2005). Computing geodesics on triangular meshes. Computers \& Graphics. 29(5):667-675.

[6]. Miranda, R. and Society, A. M. (1995). Algebraic Curves and Riemann Surfaces. Dimacs Series in Discrete Mathematics and Theoretical Comput. American Mathematical Society.

[7]. Royden H. L. (1974). The extension of regular holomorphic maps. Proceedings of the American Mathematical Society. 43(2):306-310.

[8]. Thomas A. E., Guevelou S., Di Pasquale E., Chambard A., Duval J.L., Chinesta F., Limousin V., Delgerie X., and Leroy E. (2020). Shape parametrization \& morphing in sheet-metal forming. Procedia Manufacturing. 47:702-706.

[9]. Wang Y., Chiang M.C. and Thompson P. M. (2005). Automated surface matching using mutual information applied to riemann surface structures. International Conference on Medical Image Computing and Computer-Assisted Intervention. pages 666-674. Springer.

[10]. Wang Y., Lui L. M., Gu X., Hayashi K. M., Chan T. F., Toga A. W., Thompson P. M. and Yau S.T. (2007). Brain surface conformal parameterization using riemann surface structure. IEEE Transactions on Medical Imaging. 26(6):853-865.

[11]. Wong K. W. (2020). Application of Mean Curvature Flow for Surface Parametrizations. arXiv e-prints. page arXiv:2006.07392. 